\title{
Article
}

\section{The Effects of Calculated Remnant-Like Particle Cholesterol on Incident Cardiovascular Disease: Insights from a General Chinese Population}

\author{
Yanli Chen ${ }^{1}$, Guangxiao Li ${ }^{2}$, Xiaofan Guo ${ }^{1}$, Nanxiang Ouyang ${ }^{1}$, Zhao Li ${ }^{1}$, Ning $\mathrm{Ye}^{1}$, Shasha $\mathrm{Yu}{ }^{1}$, \\ Hongmei Yang ${ }^{1}$ and Yingxian Sun ${ }^{1, * \mathbb{D}}$ \\ 1 Department of Cardiology, the First Hospital of China Medical University, Shenyang 110001, China; \\ ylchen@cmu.edu.cn (Y.C.); xfguo@cmu.edu.cn (X.G.); 20082174@cmu.edu.cn (N.O.); \\ lizhao@cmu.edu.cn (Z.L.); 2016110112@cmu.edu.cn (N.Y.); ssyu@cmu.edu.cn (S.Y.); \\ hmyang@cmu.edu.cn (H.Y.) \\ 2 Department of Medical Record Management Center, the First Affiliated Hospital of China Medical University, \\ Shenyang 110001, China; ligx1@cmu1h.com \\ * Correspondence: yxsun@cmu.edu.cn
}

\section{check for} updates

Citation: Chen, Y.; Li, G.; Guo, X.; Ouyang, N.; Li, Z.; Ye, N.; Yu, S.; Yang, H.; Sun, Y. The Effects of Calculated Remnant-Like Particle Cholesterol on Incident Cardiovascular Disease: Insights from a General Chinese Population. J. Clin. Med. 2021, 10 , 3388. https://doi.org/10.3390/ jcm10153388

Academic Editors: François Roubille and Maciej Banach

Received: 27 June 2021

Accepted: 28 July 2021

Published: 30 July 2021

Publisher's Note: MDPI stays neutral with regard to jurisdictional claims in published maps and institutional affiliations.

Copyright: (c) 2021 by the authors. Licensee MDPI, Basel, Switzerland. This article is an open access article distributed under the terms and conditions of the Creative Commons Attribution (CC BY) license (https:// creativecommons.org/licenses/by/ $4.0 /)$.

\begin{abstract}
Background: Growing evidence suggests that remnant cholesterol (RC) contributes to residual atherosclerotic cardiovascular disease (ASCVD) risk. However, the cutoff points to treat RC for reducing ASCVD are still unknown. This study aimed to investigate the relationships between RC and combined cardiovascular diseases (CVDs) in a general China cohort, with 11,956 subjects aged $\geq 35$ years. Methods: Baseline RC was estimated with the Friedewald formula for 8782 subjects. The outcome was the incidence of combined CVD, including fatal and nonfatal stroke and coronary heart disease (CHD). The Cox proportional hazards model was used to calculate hazard ratios (HRs) with $95 \%$ confidence intervals. The restricted cubic spline (RCS) model was used to evaluate the doseresponse relationship between continuous RC and the natural log of HRs. Results: After a median follow-up of 4.66 years, 431 CVD events occurred. In the Cox proportional models, participants with a high level of categorial RC had a significantly higher risk for combined CVD (HR: 1.37; 95\% CI: 1.07-1.74) and CHD (HR: 1.63; 95\% CI: 1.06-2.53), compared to those with a medium level of RC. In the stratification analyses, a high level of RC significantly increased combined CVD risk for subgroups females, age $<65$ years, noncurrent smokers, noncurrent drinkers, normal weight, renal dysfunction, and no hyperuricemia. The same trends were found for CHD among subgroups males, age $<65$ years, overweight, renal dysfunction, and no hyperuricemia; stroke among subgroup females. In RCS models, a significant linear association between RC and combined CVD and a nonlinear association between RC and CHD resulted. The risk of outcomes was relatively flat until $0.84 \mathrm{mmol} / \mathrm{L}$ of RC and increased rapidly afterwards, with an HR of 1.308 (1.102 to 1.553) for combined CVD and 1.411 (1.061 to 1.876) for CHD. Stratified analyses showed a significant nonlinear association between RC and CVD outcomes in the subgroup aged $<65$ years or the diabetes subgroup. Conclusions: In this large-scale and long-term follow-up cohort study, participants with higher RC levels had a significantly worse prognosis, especially for the subgroup aged 35-65 years or the diabetes mellitus subgroup.
\end{abstract}

Keywords: remnant cholesterol; diabetes mellitus; cardiovascular disease; dyslipidemia

\section{Introduction}

Atherosclerotic cardiovascular disease (ASCVD) is a leading cause of morbidity and mortality worldwide and remains a major public health challenge [1]. Lipid abnormalities play a central role in the pathogenesis of ASCVD [2]. Lowering plasma levels of low-density lipoprotein (LDL) cholesterol (LDL-C) has been reported as an important modality to prevent ASCVD for decades [3,4]. However, substantial residual risk remains 
despite achieving an LDL-C level as a mean of $30-40 \mathrm{mg} / \mathrm{dL}$ with statins, ezetimibe, and/or proprotein convertase subtilisin/kexin type 9 (PCSK9) inhibitors [5]. Due to the negative results of high-density lipoprotein (HDL)-raising drugs (niacin, cholesteryl ester transfer protein inhibitors), regulating other lipid components to reduce the residual risk of ASCVD has become the new focus for lipid intervention [6]. The Reduction of Cardiovascular Event Icosapent Ethyl Intervention Trial (REDUCE-IT) found that a reduction in triglycerides by $20 \%$ led to a $25 \%$ reduction in atherosclerotic cardiovascular events [7]. However, roughly one-half of the risk reduction can be explained by a reduction in remnant cholesterol (RC) [8].

In recent years, growing evidence has suggested that $\mathrm{RC}$ is a causal risk factor for cardiovascular events and all-cause mortality [9-12]. RC is the cholesterol content of triglyceride-rich lipoproteins (TRLs), which are formed when TRLs are partly depleted of triglyceride (TG) by lipoprotein lipase and are composed of very-low-density (VLDLs) and intermediate-density lipoproteins (IDLs) in the fasting state and these two lipoproteins together with chylomicron remnants in the nonfasting state [13]. The cholesterol content of remnant lipoproteins is defined as remnant-like particle cholesterol (RLP-C) [14].

However, the starting point and the target to treat RC for reducing cardiovascular diseases (CVD) are still unknown. More studies are needed to investigate the association between RC with CVD events and further determine the individual target of RC for primary and secondary prevention of CVD risk. This study aimed to investigate the relationships between RC and combined CVDs in a large general Chinese population.

\section{Materials and Methods}

\subsection{Study Design and Population}

The Northeast China Rural Cardiovascular Health Study (NCRCHS) is a communitybased prospective cohort study conducted in rural areas of Northeast China. The study design has been described previously [15]. From January 2012 to August 2013, a total of 11,956 subjects aged $\geq 35$ years were recruited as a baseline visit from three counties (Dawa, Zhangwu, and Liaoyang) in Liaoning province, using a multistage, randomly stratified cluster-sampling scheme. Detailed information was collected for each subject. In 2015 and 2017, all subjects were invited to attend two stages of follow-up. Detailed cardiovascular examination was repeated in 2015, and incident CVD events were collected in 2017-2018. Of the 11,956 subjects, 10,700 participants consented and qualified for our follow-up study. A total of 10,349 participants completed at least one follow-up visit. The study was approved by the Ethics Committee of China Medical University (Shenyang, China). Written informed consent was obtained from each participant, in accordance with the principles of the Declaration of Helsinki.

In the present analyses, we excluded participants with CVD at baseline $(n=821)$, missing a baseline lipid profile $(n=73)$, or using lipid-lowering agents at baseline $(n=248)$. Participants with ineligible values of HDL cholesterol (HDL-C), LDL-C, and TG $(>4.5 \mathrm{mmol} / \mathrm{L})$ were also excluded $(n=415)$. Data were therefore available for 8782 participants. The flow chart of the study is shown in Supplementary Figure S1 online.

\subsection{Study Variables}

At baseline, detailed information on demographic characteristics, dietary and lifestyle factors, and medical history was obtained by an interview with a standardized questionnaire. History of coronary heart disease (CHD) was defined by a self-reported history of myocardial infarction (MI) or prior coronary revascularization and electrocardiography evidence of MI at baseline and confirmed by medical records. History of stroke was similarly defined by a self-reported history of stroke at baseline and confirmed by medical records. Current use of antihypertensive medications, hypoglycemic agents, and lipid-lowering agents was self-reported. Medication use over the prior 2 weeks was verified by reviewing the medication containers that participants brought to the visit. Lipid-lowering agents included statins, bile sequestrants, fibrates, niacin, and antihyperlipidemic medications. 
Family history of hypertension, diabetes mellitus, stroke, and CHD was defined such that more than one first-degree relative was involved.

All study participants underwent a physical examination, including measurements of weight and standing height and systolic and diastolic blood pressure, as previously reported in detail [15]. Body mass index (BMI) was calculated as weight in kilograms divided by the square of height in meters. According to the World Health Organization (WHO) criteria [16], low and normal weight, overweight, and obesity were defined as BMI $<25 \mathrm{~kg} / \mathrm{m}^{2}$, BMI $25-29.9 \mathrm{~kg} / \mathrm{m}^{2}$, and BMI $\geq 30 \mathrm{~kg} / \mathrm{m}^{2}$, respectively. Blood pressure was measured three times with participants seated after at least 5 min rest using a standardized automatic electronic sphygmomanometer (HEM-907; Omron, Tokyo, Japan). Hypertension (HTN) was defined as systolic blood pressure (SBP) $\geq 140 \mathrm{~mm} \mathrm{Hg}$, diastolic blood pressure (DBP) $\geq 90 \mathrm{~mm} \mathrm{Hg}$, and/or use of antihypertensive medications. Smoking and alcohol use status was defined as "current use" or not.

\subsection{Other Variates}

Fasting blood samples were collected in the morning after at least $12 \mathrm{~h}$ fasting for all subjects. Biochemical analyses were performed automatically (Olympus AU 640, Tokyo, Japan) for the measurement of circulating concentrations of fasting blood glucose (FBG), total cholesterol (TC), LDL-C, HDL-C, TG, uric acid (UA), serum creatinine, and other routine blood biochemical indexes. As previously proposed, fasting $\mathrm{RC}$ was calculated as TC minus HDL-C minus LDL-C and expressed as mmol/L [17]. Diabetes mellitus (DM) was defined according to the WHO criterion [18]: FBG $\geq 7.0 \mathrm{mmol} / \mathrm{L}$ (126 mg/dL), self-reported physician diagnosis, or use of antidiabetic medications. Estimated glomerular filtration rate (eGFR) was calculated using the Modification of Diet in Rural Disease (MDRD) equation as previously proposed [19]. Renal dysfunction was defined as reduced eGFR $<60 \mathrm{~mL} / \mathrm{min} / 1.73 \mathrm{~m}^{2}$. Hyperuricemia was defined as serum uric acid level $>420 \mu \mathrm{mol} / \mathrm{L}(7 \mathrm{mg} / \mathrm{dL})$ in males and $>360 \mu \mathrm{mol} / \mathrm{L}(>6 \mathrm{mg} / \mathrm{dL})$ in females [20].

\subsection{Outcome Ascertainment}

The outcome of the present study was CVD incidence. Incident CVD was defined as fatal and nonfatal stroke and CHD according to adjudication by a physician panel. The specific incidences of all-cause mortality, stroke, and CHD were also determined. Hospital records or death certificates were also collected. The diagnosis was classified and coded according to the International Classification of Diseases-Tenth Revision (ICD-10). Stroke was defined following the WHO Multinational Monitoring of Trends and Determinants in Cardiovascular Disease criteria [21] as rapidly developing signs of focal or global disturbance of cerebral function lasting more than $24 \mathrm{~h}$ (unless interrupted by surgery or death) with no apparent nonvascular cause. The ICD-10 codes for stroke were I60.x-I69.x. Transient ischemic attack and chronic cerebral vascular disease were excluded. CHD was defined as a diagnosis of hospitalized angina pectoris, hospitalized MI, any coronary revascularization, or CHD death [22], and the ICD-10 codes for CHD were I20.x-25.x. All materials were independently evaluated and adjudicated by the endpoint assessment committee. The date of the first participant recruited was January 2012, and the last follow-up date was January 2018. A series of irregular follow-up visits were conducted. Follow-up time was calculated as the interval between the date of randomization and the date of death, the date of the last visit, or the last recorded clinical event of participants still alive, whichever occurred first.

\subsection{Statistical Analysis}

Data are reported as means and standard deviations for normally distributed variables or as medians for non-normally distributed variables. According to the $33.3 \%$ and $66.6 \%$ percentiles of RC level, all subjects were divided into three groups (Tertile I, Tertile II, and Tertile III). Baseline characteristics were assessed across different groups with the analysis of variance for parametric variables or Kruskal-Wallis test for nonparametric vari- 
ables. Bonferroni post-hoc analysis was performed to determine the specific demographic categories that had statistically significant differences. Nominal variables were expressed as absolute numbers and proportions and compared by a $\chi^{2}$ test or Fisher's exact test when appropriate. The correlations between continuous $\mathrm{RC}$ and adjustment variables were assessed by Pearson's or Spearman's rank correlation test as applicable.

The cumulative incidence for cardiovascular outcomes in subjects with different levels of calculated RC at baseline was evaluated by Kaplan-Meier curves and compared with the log-rank test. Cox proportional hazards models were used to calculate hazard ratios (HRs) with 95\% confidence intervals (CIs) for the associations between RC (considered as both continuous and categorical variables) and CVD events. Proportionality of hazards was assessed for each variable by using Schoenfeld residuals. Three adjustment models were found for Cox analysis. Model 1 was adjusted for age, gender, and ethnicity. Model 2 was further adjusted for current smoking, current drinking, and BMI (normal, overweight, or obesity). Model 3 was further adjusted for serum levels of triglyceride (continuous), eGFR (dichotomous), uric acid (dichotomous), DM, and HTN.

Restricted cubic spline (RCS) regression [23] was applied to flexibly model the nonlinear association between continuous RC and the natural log of HRs (lnHRs) of outcomes. The knots were placed at the 5th, 50th, and 95th percentiles. Variables in Cox Model 3 were adjusted. Stratified analyses were used to explore whether the association of RC with CVD risk varied across age and DM. Departure from linearity of the final cubic spline model was assessed using the Wald test for nonlinearity [24]. The median value of $R C$ was considered as the reference due to the skew distribution of RC. As the associations of RC and outcomes were approximately log-linear below and above their medians (50th percentile), a linear model was used to calculate HRs per standard deviation increase of RC in outcome prediction [25].

Statistical analyses were performed using SPSS (version 23.0; IBM, Chicago, IL, USA), SAS (version 9.3; Institute Inc., Cary, NC, USA), and GraphPad prism (V.8.4.2; San Diego, CA, USA). Two-sided $p<0.05$ were considered statistically significant. $p$-Values were adjusted for multiple comparisons using the Bonferroni correction.

\section{Results}

\subsection{Baseline Characteristics}

The characteristics of the participants were assessed by the tertiles of RC in Table 1. In the whole cohort of 8782 subjects $(46.4 \%$ males), the mean age was $53.2 \pm 10.4$ years. The mean BMI was $24.7 \pm 3.6 \mathrm{~kg} / \mathrm{m}^{2} ; 47.3 \%$ had HTN, and $8.4 \%$ had DM.

The mean concentration of RC was $0.8 \pm 0.4 \mathrm{mmol} / \mathrm{L}$. According to the level of RC, all participants were divided into three groups: Tertile I ( $\mathrm{RC}<0.65 \mathrm{mmol} / \mathrm{L})$, Tertile II (RC 0.65-1.00 mmol/L), and Tertile III (RC $\geq 1.00 \mathrm{mmol} / \mathrm{L}$ ). Across tertiles of RC, age, serum levels of TC, TG, uric acid, and proportion of participants with DM increased gradually (all $p<0.001$ ), whereas the level of eGFR, proportions of male, participants with HTN, and current smokers significantly declined (all $p<0.05$ ). Serum level of HDL-C and the proportion of participants with current use of alcohol were significantly higher (both $p<0.001)$ in Tertile I than those of the other two groups $(p<0.001)$. Post-hoc analysis with Bonferroni correction revealed a significantly higher age for participants in Tertile III, LDL-C level, than those in Tertile II or Tertile I $(p<0.001)$. Significantly increased levels of TC, TG, and serum uric acid and declined levels of eGFR across tertiles of RC were found. 
Table 1. Baseline characteristics by tertiles of remnant cholesterol.

\begin{tabular}{|c|c|c|c|c|c|c|c|c|}
\hline & \multirow[b]{2}{*}{$\begin{array}{c}\text { Total } \\
n=8782\end{array}$} & \multicolumn{3}{|c|}{ Remnant Cholesterol } & \multirow[b]{2}{*}{$p$-Value } & \multirow[b]{2}{*}{$p_{1}$} & \multirow[b]{2}{*}{$p_{2}$} & \multirow[b]{2}{*}{$p_{3}$} \\
\hline & & $\begin{array}{c}\text { Tertile I } \\
(n=2949)\end{array}$ & $\begin{array}{c}\text { Tertile II } \\
(n=2932)\end{array}$ & $\begin{array}{l}\text { Tertile III } \\
(n=2901)\end{array}$ & & & & \\
\hline Age (year) & $53.2 \pm 10.4$ & $52.2 \pm 10.6$ & $52.7 \pm 10.4$ & $54.7 \pm 10.0$ & $<0.001$ & 0.201 & $<0.001$ & $<0.001$ \\
\hline Male (\%) & $4075(46.4)$ & $1432(48.6)$ & $1343(45.8)$ & $1300(44.8)$ & 0.012 & & & \\
\hline Ethnicity of Han (\%) & $8277(94.2)$ & $2664(90.3)$ & $2820(96.2)$ & $2793(96.3)$ & $<0.001$ & & & \\
\hline Current smoking (\%) & $3118(35.5)$ & $1121(38.0)$ & $1022(34.9)$ & $975(33.6)$ & 0.001 & & & \\
\hline $\begin{array}{l}\text { Current drinking } \\
\text { alcohol (\%) }\end{array}$ & $1992(22.7)$ & $755(25.6)$ & $619(21.1)$ & $618(21.3)$ & $<0.001$ & & & \\
\hline \multicolumn{9}{|l|}{ Physical activity } \\
\hline Low & $2964(33.8)$ & $819(27.8)$ & $1033(35.2)$ & $1112(38.3)$ & & & & \\
\hline Medium & $1652(18.8)$ & $566(19.2)$ & $581(19.8)$ & $505(17.4)$ & $<0.001$ & & & \\
\hline High & $4091(46.6)$ & $1534(52.0)$ & $1290(44.0)$ & $1267(44.0)$ & & & & \\
\hline HTN (\%) & $4154(47.3)$ & $1141(38.7)$ & $965(32.9)$ & $911(31.4)$ & $<0.001$ & & & \\
\hline $\mathrm{DM}(\%)$ & $738(8.4)$ & $201(6.8)$ & $212(7.2)$ & $325(11.2)$ & $<0.001$ & & & \\
\hline $\mathrm{BMI}\left(\mathrm{kg} / \mathrm{m}^{2}\right)$ & $24.7 \pm 3.6$ & $24.7 \pm 3.8$ & $24.4 \pm 3.6$ & $24.8 \pm 3.5$ & $<0.001$ & 0.003 & 0.866 & $<0.001$ \\
\hline $\mathrm{SBP}(\mathrm{mmHg})$ & $140.5 \pm 22.7$ & $144.0 \pm 24.3$ & $137.1 \pm 21.6$ & $140.3 \pm 21.5$ & $<0.001$ & $<0.001$ & $<0.001$ & $<0.001$ \\
\hline $\mathrm{DBP}(\mathrm{mmHg})$ & $81.6 \pm 11.5$ & $81.1 \pm 11.8$ & $81.1 \pm 11.5$ & $82.6 \pm 11.3$ & $<0.001$ & 1.0 & $<0.001$ & $<0.001$ \\
\hline FBG $(\mathrm{mmol} / \mathrm{L})$ & $5.8 \pm 1.5$ & $5.6 \pm 1.4$ & $5.8 \pm 1.7$ & $5.8 \pm 1.3$ & $<0.001$ & 0.043 & $<0.001$ & $<0.001$ \\
\hline $\mathrm{TC}(\mathrm{mmol} / \mathrm{L})$ & $5.2 \pm 1.0$ & $4.7 \pm 0.9$ & $5.0 \pm 0.8$ & $5.8 \pm 1.0$ & $<0.001$ & $<0.001$ & $<0.001$ & $<0.001$ \\
\hline $\mathrm{TG}(\mathrm{mmol} / \mathrm{L})$ & $1.4 \pm 0.8$ & $1.1 \pm 0.5$ & $1.3 \pm 0.7$ & $1.8 \pm 0.8$ & $<0.001$ & $<0.001$ & $<0.001$ & $<0.001$ \\
\hline $\mathrm{HDL}-\mathrm{C}(\mathrm{mmol} / \mathrm{L})$ & $1.4 \pm 0.3$ & $1.6 \pm 0.4$ & $1.3 \pm 0.3$ & $1.3 \pm 0.3$ & $<0.001$ & $<0.001$ & $<0.001$ & 0.626 \\
\hline LDL-C (mmol/L) & $2.9 \pm 0.8$ & $2.8 \pm 0.8$ & $2.8 \pm 0.7$ & $3.1 \pm 0.8$ & $<0.001$ & 0.429 & $<0.001$ & $<0.001$ \\
\hline $\begin{array}{c}\text { Estimated eGFR } \\
\left(\mathrm{mL} / \mathrm{min} / 1.73 \mathrm{~m}^{2}\right)\end{array}$ & $94.1 \pm 15.1$ & $101.6 \pm 13.2$ & $92.3 \pm 14.3$ & $88.2 \pm 14.4$ & $<0.001$ & $<0.001$ & $<0.001$ & $<0.001$ \\
\hline $\begin{array}{l}\text { Serum uric acid } \\
(\mu \mathrm{mol} / \mathrm{L})\end{array}$ & $285.6 \pm 81.5$ & $260.7 \pm 73.8$ & $284.8 \pm 79.5$ & $290.4 \pm 78.5$ & $<0.001$ & $<0.001$ & $<0.001$ & $<0.001$ \\
\hline $\mathrm{RC}(\mathrm{mmol} / \mathrm{L})$ & $0.83 \pm 0.44$ & $0.36 \pm 0.16$ & $0.84 \pm 0.10$ & $1.32 \pm 0.30$ & $<0.001$ & $<0.001$ & $<0.001$ & $<0.001$ \\
\hline
\end{tabular}

BMI: body mass index; DBP: diastolic blood pressure; DM: diabetes mellitus; FPG: fasting plasma glucose; GFR: glomerular filtration rate; HDL-C: high-density lipoprotein cholesterol; HTN: hypertension; LDL-C: low-density lipoprotein cholesterol; RC: remnant cholesterol; SBP: systolic blood pressure; SD: standard deviation; TC: total cholesterol; TG: triglyceride. Data are expressed as mean \pm SD or as $n(\%) . p$-Value: statistical significance among three groups; $p_{1}, p_{2}$, and $p_{3}$ : respective statistical significance with Bonferroni correction for measurement data between Tertile I and Tertile II, Tertile I and Tertile III, and between Tertile II and Tertile III.

\subsection{Survival Analyses for Different Levels of RC}

After the median follow-up time of 4.66 years, a total of 431 CVD events occurred in the studied population (293 stroke cases, 150 CHD cases, and 71 MI cases), including 148 fatal CVD cases. The Kaplan-Meier curves for each endpoint in participants with different levels of RC are shown in Figure 1. Participants with high level of RC (Tertile III) had a significantly higher cumulative incidences of combined CVD $(p=0.0019)$, CHD $(p=0.0101)$, stroke $(p=0.0448)$, and fatal CVD $(p=0.0465)$ compared to those with a medium level of RC (Tertile II).

Table 2 shows the multivariable-adjusted HRs for the incidence of outcomes by $\mathrm{RC}$ concentration. In the categorial analysis of RC, risks for combined CVD (HR: 1.37; 95\% CI: 1.07-1.74) and CHD (HR: 1.63; 95\% CI: 1.06-2.53) were significantly higher among participants in Tertile III, compared with those in Tertile II after full adjustment. In the continuous analysis of RC, a high level of RC significantly increased $28 \%$ risk of combined CVD (HR: 1.28; 95\% CI: 1.02-1.62) and 51\% risk of fatal CVD (HR: 1.51; 95\% CI: 1.05-2.17) after full adjustment. Significantly higher stroke risk was found for participants in Tertile III after adjustment for Model 1 (HR: 1.31; 95\% CI: 1.03-1.67) and Model 2 (HR: 1.30; $95 \%$ CI: 1.02-1.67), but the significance disappeared for Model 3. The results were similar when the intensity of physical activity was additionally adjusted. 


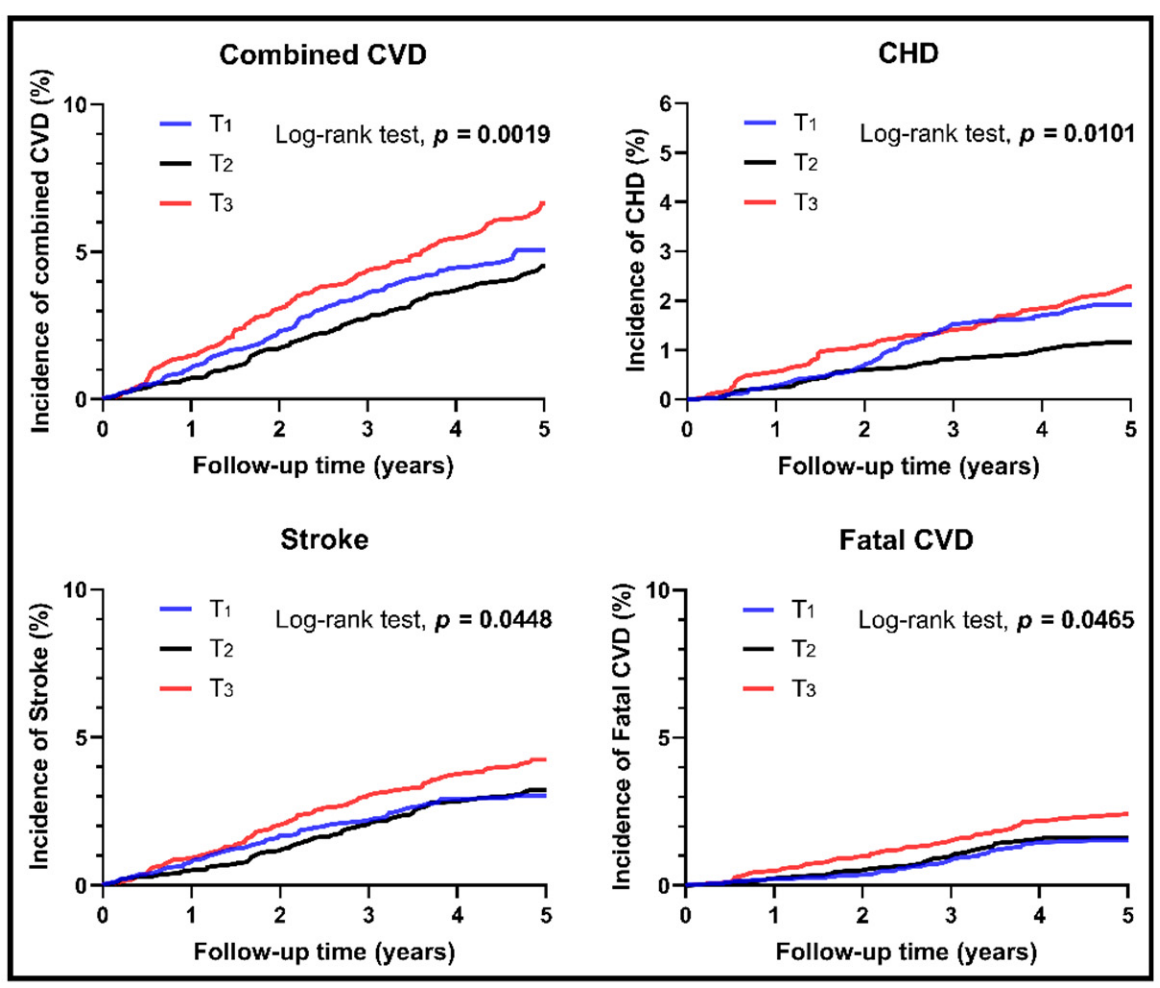

Figure 1. Unadjusted Kaplan-Meier curves for incident cardiovascular events stratified by different levels of remnant cholesterol. The whole cohort was divided into three groups due to different levels of remnant cholesterol. Low level: Tertile I; Medium level: Tertile II; High level: Tertile III. CHD: coronary heart disease; CVD: cardiovascular disease.

Table 2. Multivariate-adjusted hazard ratios and 95\% confidence intervals for cardiovascular outcomes associated with baseline remnant cholesterol.

\begin{tabular}{|c|c|c|c|c|}
\hline & $\mathrm{RC} \mathrm{Q}_{1}$ & $\mathrm{RC} \mathrm{Q}_{2}$ & $\mathrm{RC} \mathbf{Q}_{3}$ & RC Continuous (1-SD $\left.{ }^{+}\right)$ \\
\hline \multicolumn{5}{|c|}{ Combined CVD } \\
\hline$n / N$ & $139 / 2191$ & $117 / 2932$ & $175 / 2901$ & $431 / 8782$ \\
\hline Model 1 & $1.23(0.96-1.57)$ & 1.00 (ref) & $1.49(1.18-1.89) * *$ & $1.33(1.09-1.63) * *$ \\
\hline Model 2 & $1.22(0.95-1.57)$ & 1.00 (ref) & $1.49(1.18-1.89)^{* *}$ & $1.32(1.08-1.62)^{* *}$ \\
\hline Model 3 & $1.16(0.90-1.50)$ & 1.00 (ref) & $1.37(1.07-1.74) *$ & $1.28(1.02-1.62) *$ \\
\hline \multicolumn{5}{|l|}{ CHD } \\
\hline$n / N$ & $55 / 2191$ & $34 / 2932$ & $61 / 2901$ & $150 / 8782$ \\
\hline Model 1 & $1.77(1.15-2.74)^{*}$ & 1.00 (ref) & $1.71(1.12-2.61) *$ & $1.17(0.82-1.67)$ \\
\hline Model 2 & $1.74(1.12-2.70)$ * & 1.00 (ref) & $1.71(1.12-2.61) *$ & $1.16(0.81-1.66)$ \\
\hline Model 3 & $1.68(1.08-2.62)$ * & 1.00 (ref) & $1.63(1.06-2.53)$ * & $1.15(0.76-1.74)$ \\
\hline \multicolumn{5}{|l|}{ Stroke } \\
\hline$n / N$ & $88 / 2191$ & $87 / 2932$ & $118 / 2901$ & $293 / 8782$ \\
\hline Model 1 & $1.04(0.77-1.40)$ & 1.00 (ref) & $1.30(0.98-1.72)$ & $1.31(1.03-1.67) *$ \\
\hline Model 2 & $1.04(0.77-1.41)$ & 1.00 (ref) & $1.31(0.98-1.73)$ & $1.30(1.02-1.67)$ * \\
\hline Model 3 & $0.99(0.73-1.35)$ & 1.00 (ref) & $1.19(0.89-1.59)$ & $1.25(0.94-1.66)$ \\
\hline \multicolumn{5}{|c|}{ Fatal CVD } \\
\hline$n / N$ & $41 / 2191$ & $44 / 2932$ & $63 / 2901$ & $148 / 8782$ \\
\hline Model 1 & $0.97(0.64-1.49)$ & 1.00 (ref) & $1.394(0.95-2.05)$ & $1.44(1.05-1.97)$ * \\
\hline Model 2 & $0.95(0.62-1.46)$ & 1.00 (ref) & $1.421(0.97-2.09)$ & $1.47(1.07-2.01) *$ \\
\hline Model 3 & $0.90(0.58-1.39)$ & 1.00 (ref) & $1.37(0.92-2.05)$ & $1.51(1.05-2.17)$ * \\
\hline
\end{tabular}

CHD: coronary heart disease; CVD: cardiovascular disease; RC: remnant cholesterol. Model 1: adjusted for age ( $<65$ years vs. $\geq 65$ years), sex, and ethnicity (Han or not). Model 2: adjusted for factors in Model 1 and smoking status, drinking status, and body mass index (normal, overweight, obesity). Model 3: adjusted for factors in Model 2 and estimated glomerular filtration rate $\left(<60 \mathrm{~mL} / \mathrm{min} / 1.73 \mathrm{~m}{ }^{2}\right.$ vs. $\geq 60 \mathrm{~mL} / \mathrm{min} / 1.73 \mathrm{~m}^{2}$ ), diabetes mellitus (yes or no), hypertension (yes or no), triglyceride (continuous), and hyperuricemia (yes or no). ${ }^{\dagger} \mathrm{HR}$ for continuous $1-\mathrm{SD}$ increment. ${ }^{*} p<0.05 ;{ }^{* *} p<0.01$. 


\subsection{Stratification Analyses}

The relationships between RC and outcomes were evaluated among different RC categories (Tertile II as reference) and stratified with sex, age ( $<65$ years vs. $\geq 65$ years), smoking status (current smoker or not), drinking status (current drinker or not), BMI subgroups (normal, overweight, obesity), DM (yes or no), HTN (yes or no), renal dysfunction (eGFR $<60 \mathrm{~mL} / \mathrm{min} / 1.73 \mathrm{~m}^{2}$ vs. eGFR $\geq 60 \mathrm{~mL} / \mathrm{min} / 1.73 \mathrm{~m}^{2}$ ), and hyperuricemia (yes or no). Due to the small number of other nationalities except Han, there were no subgroup comparisons for nationality.

After full adjustment of other factors, subjects with a high level of RC (Tertile III) had a significantly higher incidence of CVD than those with a medium level of RC (Tertile II) in the following subgroups (Figure 2): females (HR: 1.515, 95\%CI: 1.043-2.201), subjects aged < 65 years (HR: 1.547, 95\%CI: 1.127-2.123), noncurrent smokers (HR: $1.482,95 \% \mathrm{CI}$ : 1.064-2.062), noncurrent drinkers (HR: $1.431,95 \% \mathrm{CI}: 1.079-1.898)$, subjects with normal BMI (HR: 1.486, 95\%CI: 1.046-2.110), renal dysfunction (HR: 1.357, 95\%CI: 1.050-1.752), and normal uric acid (HR: 1.439, 95\%CI: 1.101-1.880). The same trends were found for CHD among subgroups: aged $\leq 65$ years, overweight subgroup, renal dysfunction, and normal uric acid (Supplementary Figure S2). For participants in Tertile III, males had a significantly higher risk for CHD (HR: 2.170, 95\%CI: 1.038-4.537), and females had more probability of stroke (HR: 1.643, 95\%CI: 1.010-2.686) compared to those in Tertile II (Supplementary Figure S3). There were no significant interactions between subgroups and $\mathrm{RC}$ on the incidence of the above outcomes (all $p>0.05$ ).

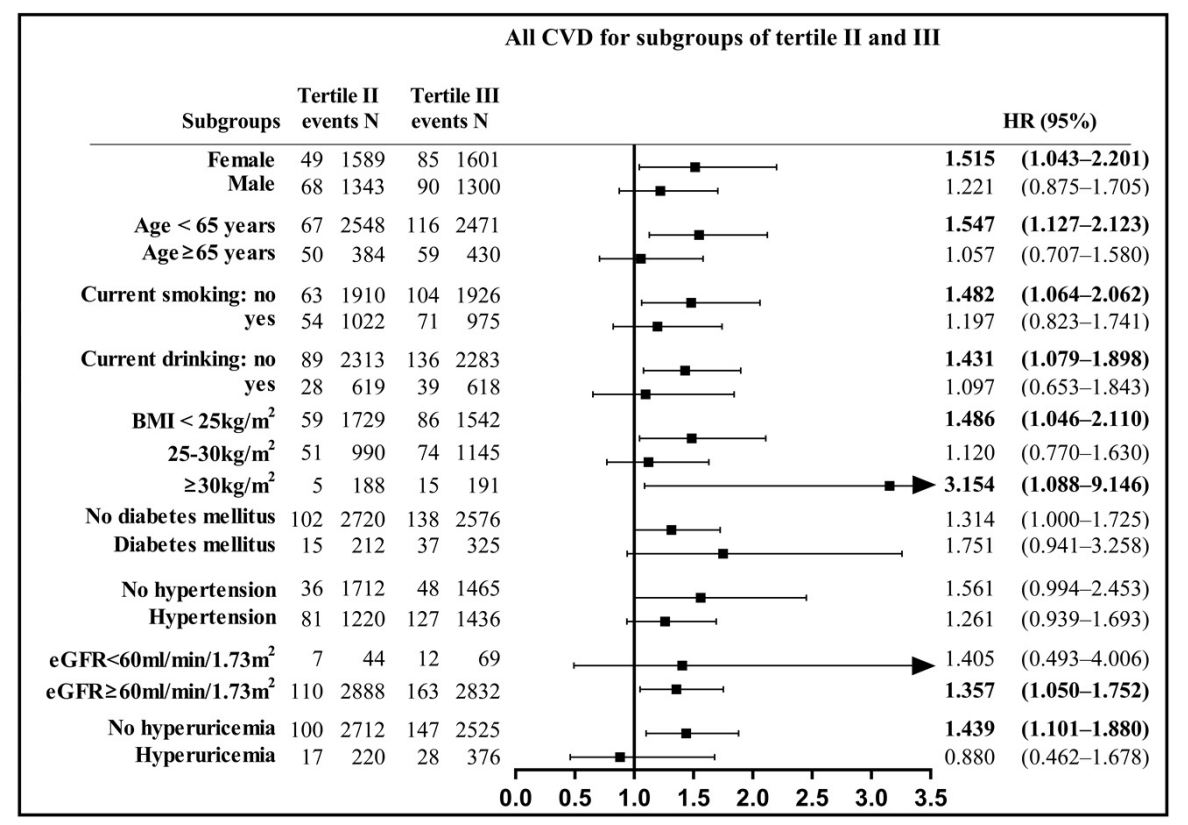

Figure 2. Remnant cholesterol (Tertile II and Tertile III) in relation to combined cardiovascular disease (CVD) for different subgroups. Model adjusted for age, sex, ethnicity, body mass index, smoking status, drinking status, hypertension, diabetes mellitus, estimated glomerular filtration rate, hyperuricemia, and continuous triglyceride. BMI: body mass index; CI: confidence interval; CVD: cardiovascular disease; eGFR: estimated glomerular filtration rate; HR: hazard ratio; RC: remnant cholesterol.

Compared with the reference (Tertile II), a low level of RC (Tertile I) also increased the risk for CHD in subgroups aged $<65$ years, no current smoking, no current drinking, BMI $25-30 \mathrm{~kg} / \mathrm{m}^{2}$, renal dysfunction, and normal uric acid (Supplementary Figure S4). No significant differences were found for fatal CVD among participants in Tertile III and combined CVD, stroke, and fatal CVD among participants in Tertile I. No signif- 
icant interactions between subgroups and $\mathrm{RC}$ were found on the incidence of outcomes (all $p>0.05)$.

\subsection{Dose-Response Analyses of $R C$ with Cardiovascular Outcomes}

Dose-response analyses were implemented with RCS to investigate the optimal level of RC for each outcome (Figure 3). Multivariable-adjusted RCS analyses showed significant overall associations between RC and combined CVD ( $\left.p_{\text {overall }}=0.0324\right)$ or CHD $\left(p_{\text {overall }}=0.0398\right)$ for the whole cohort. A significant linear relationship $\left(p_{\text {linear }}=0.0225\right)$ between RC and combined CVD and a nonlinear relationship ( $\left.p_{\text {nonlinear }}=0.0221\right)$ between $\mathrm{RC}$ and CHD was found for all participants. The risk of combined CVD was relatively flat until around $0.84 \mathrm{mmol} / \mathrm{L}(32.76 \mathrm{mg} / \mathrm{dL})$ of $\mathrm{RC}$ and then started to increase rapidly afterwards. For participants with a higher level of RC than $0.84 \mathrm{mmol} / \mathrm{L}$, the HR of combined CVD and CHD per standard deviation was 1.308 (1.102 to 1.553) and 1.411 (1.061 to 1.876$)$, respectively.
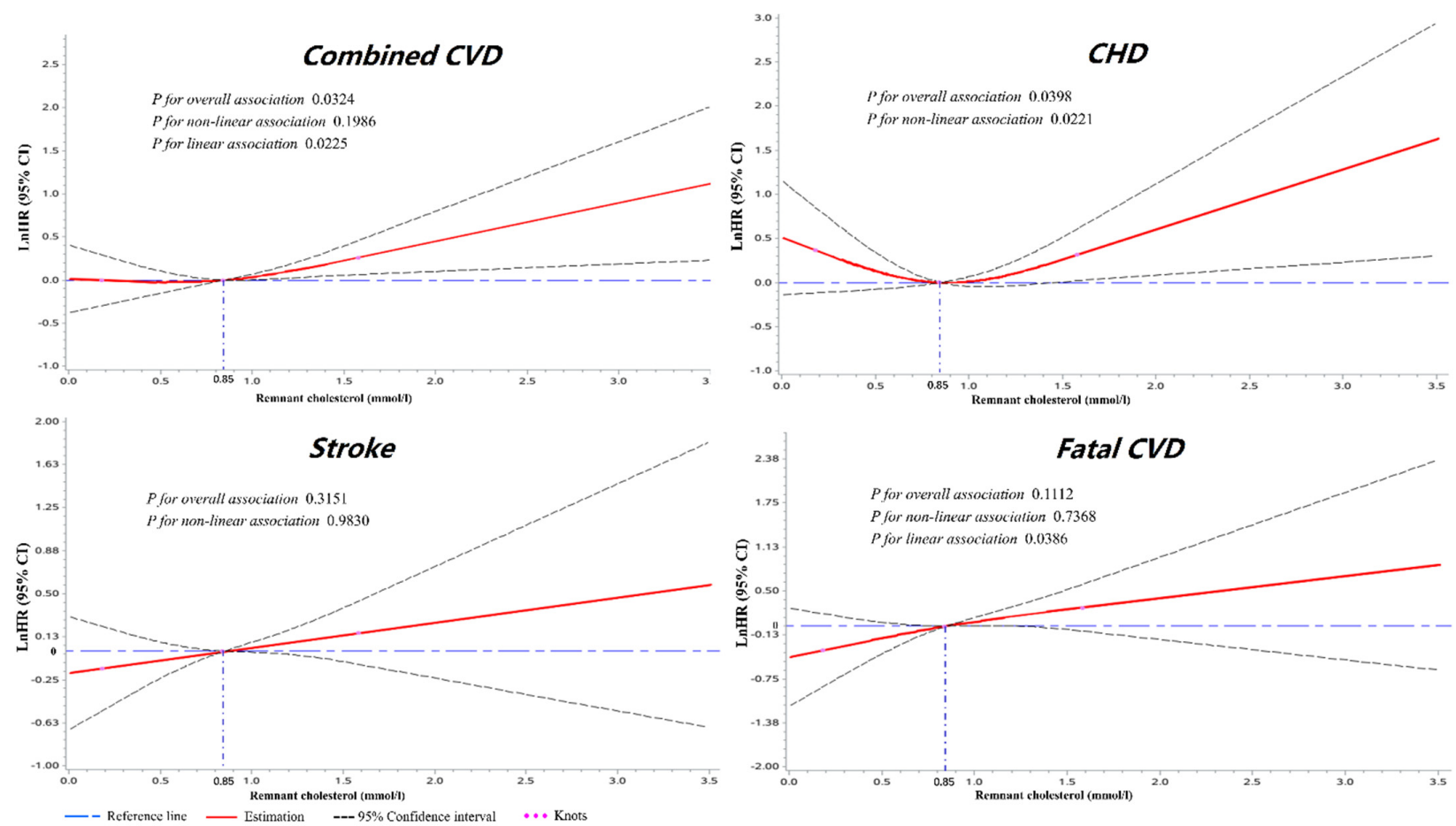

Figure 3. The dose-response associations between RC and cardiovascular outcomes for the whole cohort. Restricted cubic splines displaying the lnHRs of cardiovascular events with $95 \%$ confidence intervals according to the serum level of remnant cholesterol. Reference set to median $(0.84 \mathrm{mmol} / \mathrm{L}$ ). Knots located at $0.18,0.84$, and $1.58 \mathrm{mmol} / \mathrm{L}$ (at the 5 th, 50 th, and $95 \mathrm{th}$ percentiles). Adjusted for age, sex, ethnicity, body mass index, smoking status, drinking status, hypertension, diabetes mellitus, estimated glomerular filtration rate, hyperuricemia, and continuous triglyceride. CHD: coronary heart disease; CVD: cardiovascular disease; lnHRs: natural log of hazard ratios; RC: remnant cholesterol.

Nonlinear associations between RC and lnHRs for outcomes stratified by subgroups ( $<65$ years vs. $\geq 65$ years; DM or not) are shown in Figure 4 . Significant nonlinearity association and "J"-shape curves were shown for RC and combined CVD $\left(p_{\text {nonlinear }}=0.0059\right)$ or CHD ( $\left.p_{\text {nonlinear }}=0.0002\right)$ in participants with an age $<65$ years (Figure 4A). Meanwhile, a linear association was found between RC and $\operatorname{lnHR}$ for fatal CVD $\left(p_{\text {linear }}=0.0104\right)$. A $50.7 \%$ increase risk for combined CVD, a $57.0 \%$ increase risk for CHD, and a $2.0 \%$ increase risk for fatal CVD were found for participants under 65 years with a serum level of $\mathrm{RC}>0.83 \mathrm{mmol} / \mathrm{L}$. The same trend was found for participants with DM and a serum level of RC $>0.95 \mathrm{mmol} / \mathrm{L}$ (Figure 4B). Some significant associations were also found among other subgroups (Supplementary Figures S5-S7). 


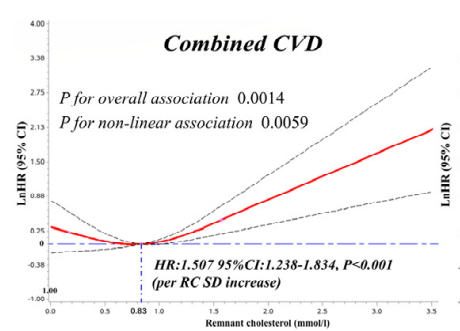

A

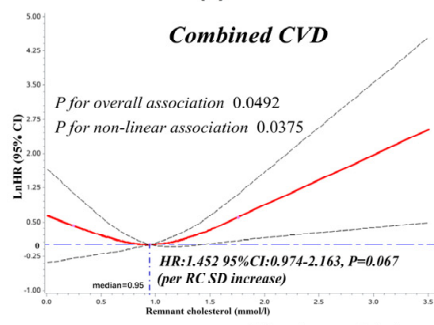

B

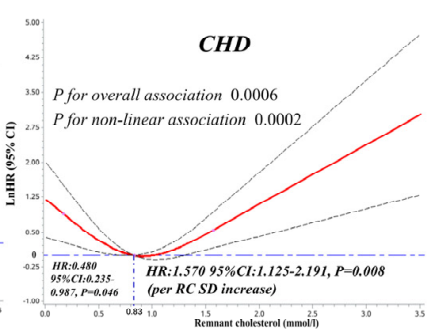

(2)

CHD

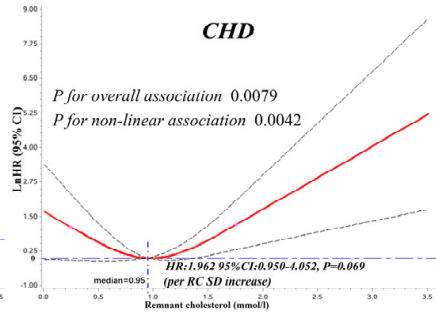

Knots

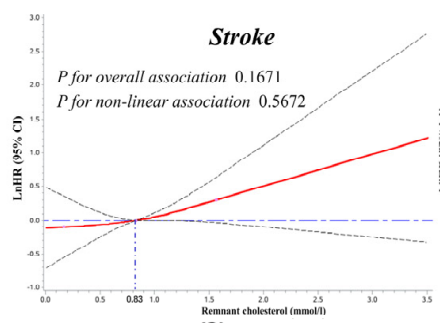

(3)

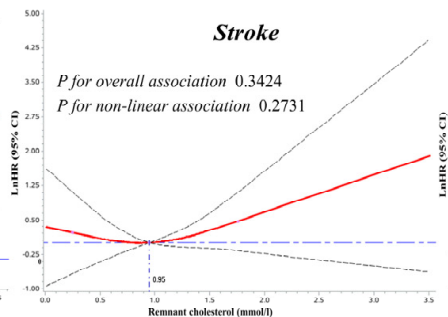

(3)

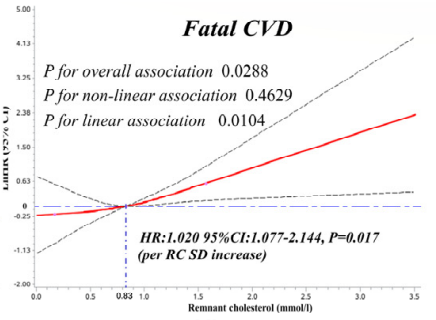

(4)

Fatal CHD

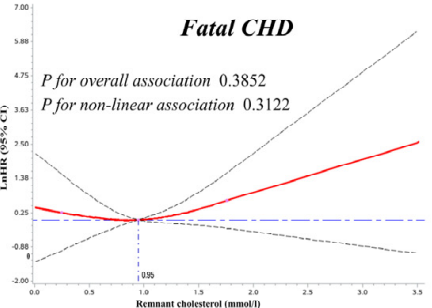

(4)

Figure 4. Dose-response associations between RC and cardiovascular outcomes for subgroups aged $<65$ years and DM. Restricted cubic splines displaying the lnHRs of cardiovascular events among participants aged $<65$ years (A(1-4)) and diabetes (B(1-4)) with 95\% confidence intervals according to the serum level of remnant cholesterol. Reference set to medians $(0.83 \mathrm{mmol} / \mathrm{L}$ for subgroups aged $<65$ years, $0.95 \mathrm{mmol} / \mathrm{L}$ for the diabetes mellitus subgroup). Knots located at the 5th, 50th, and 95th percentiles of remnant cholesterol (subgroup aged < 65 years: 0.17, 0.83, and 1.57 mmol/L; DM: 0.25 , 0.95 , and $1.76 \mathrm{mmol} / \mathrm{L}$ ). Adjusted for age, sex, ethnicity, body mass index, smoking status, drinking status, hypertension, $\mathrm{DM}$, estimated glomerular filtration rate, hyperuricemia, and continuous triglyceride. CHD, coronary heart disease; CVD, cardiovascular disease; DM, diabetes mellitus; HR, hazard ratio; lnHRs, natural log of hazard ratios; RC, remnant cholesterol; SD, standard deviation.

\section{Discussion}

The current study of a general Chinese population confirmed an independent relationship between calculated RC and the incidence of CVD. The Kaplan-Meier curves explored significantly higher cumulative incidences of combined CVD, CHD, stroke, and fatal CVD for participants with a high level of RC than those with a medium level of RC. Cox models confirmed a $37 \%$ increased risk for combined CVD and a $63 \%$ increased risk for CHD in the categorial analysis and a $28 \%$ increased risk of combined CVD and a $51 \%$ increased risk of fatal CVD in the continuous analysis of RC. A significantly higher risk of stroke was also found for participants with an RC level of Tertile III after part adjustment. Further doseresponse association analyses and subgroup analyses elucidated significant associations between continuous RC and the incidence of combined CVD and CHD not only for the whole population but also for the subgroup aged $<65$ years or the DM subgroup.

RC refers to the cholesterol content of TRLs, which is composed of chylomicron remnants, VLDL, and IDL. A recent study showed a close relationship between RC and coronary atheroma progression independent of conventional lipid parameters [11]. A growing number of population studies, epidemiological, and genetic evidence suggests that high concentrations of $\mathrm{RC}$ are closely associated with a high risk of ischemic heart disease $[9,26,27]$, MI $[28,29]$, and all-cause mortality [10,30]. A cardiovascular benefit of statin therapy, independently of LDL-C reduction, was suggested in reducing TRL-C levels among those with high TRL-C levels [31].

The onset age of CVD is getting younger and younger worldwide [2,32]. Dyslipidemia is a prominently traditional risk factor in the development of early-onset CVD [33,34]. Early identification and modification of atherogenic dyslipidemia can improve primary and secondary prevention of CVD outcomes. The ideal level of lipids is very important in reducing the risk of early-onset cardiovascular-related death $[35,36]$. Lifestyle modification at the age of 20 years could change the odds of atherosclerosis 30 years later [37]. The currently employed CVD risk assessment tools are heavily age weighted and have 
been shown to underestimate CVD risk in young to middle-aged populations [38-40]. However, the optimal RC level for preventing CVD in young to middle-aged populations has not yet been reported. The present study suggested increased risks of CVD, CHD, and fatal CVD for participants 35 to 65 years of age and an RC level $>0.83 \mathrm{mmol} / \mathrm{L}$. This may contribute to risk stratification for dyslipidemia and constructing further treatment strategies to prevent CVD in young to middle-aged populations.

DM confers at least a two to threefold excess risk for ASCVD [41]. Data have revealed that serum $\mathrm{RC}$ concentrations are elevated in patients with $\mathrm{DM}$ or pre-DM and can increase the risk for CHD and future coronary outcomes [42-44]. This suggests RC as a possible treatment target for patients with impaired glucose metabolism [17]. A baseline RC level $\geq 30 \mathrm{mg} / \mathrm{dL}(0.78 \mathrm{mmol} / \mathrm{L})$ identifies a high risk of major adverse cardiovascular events for individuals with type $2 \mathrm{DM}$ (T2DM) or more than three CVD risk factors [45]. Additionally, the risk is independent of whether LDL-C levels are on target at $\leq 100 \mathrm{mg} / \mathrm{dL}$ $(2.59 \mathrm{mmol} / \mathrm{L})$ or not. For patients with T2DM who underwent previous percutaneous coronary stents, a baseline RLP-C level at $0.505 \mathrm{mmol} / \mathrm{L}$ was identified as the optimal cutoff point to predict in-stent restenosis [46]. The lipid level for participants with DM should be lower than those without DM for preventing ASCVD. Several guidelines proposed a stratification of cardiovascular risk among people with DM and recommend an intensive therapy for dyslipidemia in DM [47,48]. However, the target for regulating RC concentration is still not assured among Chinese populations with DM. In this study, the incidence of combined CVD and CHD significantly increased for general Chinese participants with a T2DM and serum level of RC $>0.95 \mathrm{mmol} / \mathrm{L}$ (Figure 4). It follows that RC is another intervening target to reduce CVD except LDL-C.

The mechanism behind this cardiovascular benefit for RC is not fully understood. There is a complex link between RC, inflammation, and CVD events [49]. Balling evidence revealed that one-third of TC in plasma was present in RC by using direct measurements [50]. High levels of RC can cause a variety of proatherogenic effects, including monocyte activation, upregulation of proinflammatory cytokines, and increased prothrombotic factors production [26,51]. Remnant lipoproteins can enter and get trapped in the intima of the arterial wall, promoting endothelial dysfunction and inflammation through increased secretion of various cytokines and adhesion molecules [52]. Without oxidative modification by macrophages [53], RC is accumulated in the arterial wall and may play a causal role in the development of atherosclerosis and ultimately ASCVD [26,27]. RC also accelerates the onset of endothelial progenitor cells senescence via increased oxidative stress and induces endothelial dysfunction by inhibiting nitric oxide production [54]. Autopsy case analysis showed coronary atherosclerosis in the young ( $<40$ years), commonly exhibiting eccentric plaques with associated inflammation [55]. The atherosclerotic plaques of younger patients are rich in foam cells, which reduce plaque stability and induce earlyonset ACS [56]. Mazzone reviewed the important roles of diabetic dyslipidemia (beyond the LDL cholesterol level) and inflammation for accelerating vascular injury and increasing the rates of CVD in T2DM patients [57].

The present study demonstrated that high levels of RC were significant predictors of increased risk of CVD events, especially for the 35- to 65-years-old population and DM patients. This suggests that assessing RC levels in these populations might be likely to have clinical utility in terms of CVD risk stratification and future intervention. Further investigations into RC lowering interventions to reduce residual ASCVD risk are necessary. This study had several limitations. Firstly, RC was calculated with the Friedewald formula but not measured directly. A simple and widely available assay is needed to be developed to measure the cholesterol content of RC. Secondly, the effect of different constructions of diet on RC concentration is lacking. Finally, this was a study among a general Chinese population. Studies of RC in other countries with different diet cultures need further investigation. 


\section{Conclusions}

In conclusion, in this large-scale population-based study, we found a high level of $\mathrm{RC}$ significantly related to a worse prognosis. For the first time, we revealed that the risk of CVD significantly increased after the median values of RC for young to middle-aged populations $(0.83 \mathrm{mmol} / \mathrm{L})$ and the DM subgroup $(0.95 \mathrm{mmol} / \mathrm{L})$. RC as a causative risk factor for CVD events deserves further attention and may constitute a prominent target for interventions to reduce vascular risk after LDL cholesterol lowering, especially for young to middle-aged populations or participants with diabetes.

Supplementary Materials: The following are available online at https:/ / www.mdpi.com/article/10 .3390 /jcm10153388/s1, Figure S1: Study flow chart, Figure S2: Remnant cholesterol (tertile II and tertile III) in relation to coronary heart disease for different subgroups, Figure S3: Remnant cholesterol (tertile II and tertile III) in relation to stroke for different subgroups, Figure S4: Remnant cholesterol (tertile I and tertile II) in relation to coronary heart disease for different subgroups, Figures S5-S7: Significant dose-response associations between remnant cholesterol and cardiovascular outcomes for some subgroups.

Author Contributions: Data curation, Y.C., G.L. and S.Y.; Formal analysis, Y.C. and G.L.; Funding acquisition, Y.S.; Investigation, Y.S.; Methodology, Y.C., G.L. and X.G.; Project administration, Y.C., Z.L., and Y.S.; Resources, X.G., N.O., Z.L., S.Y. and H.Y.; Software, G.L.; Supervision, Y.S.; Validation, Y.C., N.O. and N.Y.; Visualization, G.L.; Writing-original draft preparation, Y.C., N.Y.; Writing -review and editing, Y.C., X.G. and H.Y. All authors have read and agreed to the published version of the manuscript.

Funding: Professor YXS received funding from the National Key Research and Development Program from the Ministry of Science and Technology of China (Project Grant Number 2018YFC1312400, Subproject Grant Number 2018YFC1312403).

Institutional Review Board Statement: The study was approved by the institutional ethics review committee of the First Affiliated Hospital of China Medical University (AF-SOP-07-1.0-01). All patients were given the opportunity to withdraw their participation using an opt-out procedure.

Informed Consent Statement: Not applicable.

Data Availability Statement: All data generated or analyzed during this study are included in this published article (and its Supplementary Information files). The datasets generated during and/or analyzed during the current study are not publicly available due to the lack of a specific patients' consent but are made available by the corresponding author based on a reasonable request.

Acknowledgments: The authors thank the staff and participants of the NCRCHS study for their important contributions. This study was funded by the National Key Research and Development Program from the Ministry of Science and Technology of China (Project Grant Number 2018YFC1312400, Subproject Grant Number 2018YFC1312403).

Conflicts of Interest: The authors declare that they have no competing interests.

\section{References}

1. Yang, G.; Wang, Y.; Zeng, Y.; Gao, G.F.; Liang, X.; Zhou, M.; Wan, X.; Yu, S.; Jiang, Y.; Naghavi, M.; et al. Rapid health transition in China, 1990-2010: Findings from the Global Burden of Disease Study 2010. Lancet 2013, 381, 1987-2015. [CrossRef]

2. Yusuf, S.; Hawken, S.; Ounpuu, S.; Dans, T.; Avezum, A.; Lanas, F.; McQueen, M.; Budaj, A.; Pais, P.; Varigos, J.; et al. Interheart Study Investigators. Effect of potentially modifiable risk factors associated with myocardial infarction in 52 countries (the INTERHEART study): Case-control study. Lancet 2004, 364, 937-952. [CrossRef]

3. Cholesterol Treatment Trialists' (CTT) Collaboration; Baigent, C.; Blackwell, L.; Emberson, J.; Holland, L.E.; Reith, C.; Bhala, N.; Peto, R.; Barnes, E.H.; Keech, A.; et al. Efficacy and safety of more intensive lowering of ldl cholesterol: A meta-analysis of data from 170,000 participants in 26 randomised trials. Lancet 2010, 376, 1670-1681.

4. Silverman, M.G.; Ference, B.A.; Im, K.; Wiviott, S.D.; Giugliano, R.P.; Grundy, S.M.; Braunwald, E.; Sabatine, M.S. Association between lowering ldl-c and cardiovascular risk reduction among different therapeutic interventions: A systematic review and meta-analysis. JAMA 2016, 316, 1289-1297. [CrossRef]

5. Schwartz, G.G.; Steg, P.G.; Szarek, M.; Bhatt, D.L.; Bittner, V.A.; Diaz, R.; Edelberg, J.M.; Goodman, S.G.; Hanotin, C.; Harrington, R.A.; et al. Alirocumab and Cardiovascular Outcomes after Acute Coronary Syndrome. N. Engl. J. Med. 2018, 379, 2097-2107. [CrossRef] 
6. Sandesara, P.B.; Virani, S.S.; Fazio, S.; Shapiro, M.D. The Forgotten Lipids: Triglycerides, Remnant Cholesterol, and Atherosclerotic Cardiovascular Disease Risk. Endocr. Rev. 2019, 40, 537-557. [CrossRef] [PubMed]

7. Bhatt, D.L.; Steg, P.G.; Miller, M.; Brinton, E.A.; Jacobson, T.A.; Ketchum, S.B.; Doyle, R.T.; Juliano, R.A.; Jiao, L.; Granowitz, C.; et al. Cardiovascular Risk Reduction with Icosapent Ethyl for Hypertriglyceridemia. N. Engl. J. Med. 2019, 380, 11-22. [CrossRef] [PubMed]

8. Langsted, A.; Madsen, C.M.; Nordestgaard, B.G. Contribution of remnant cholesterol to cardiovascular risk. J. Intern. Med. 2020, 288, 116-127. [CrossRef] [PubMed]

9. Varbo, A.; Benn, M.; Tybjærg-Hansen, A.; Jørgensen, A.B.; Frikke-Schmidt, R.; Nordestgaard, B.G. Remnant Cholesterol as a Causal Risk Factor for Ischemic Heart Disease. J. Am. Coll. Cardiol. 2013, 61, 427-436. [CrossRef]

10. Varbo, A.; Freiberg, J.J.; Nordestgaard, B.G. Extreme Nonfasting Remnant Cholesterol vs Extreme LDL Cholesterol as Contributors to Cardiovascular Disease and All-Cause Mortality in 90000 Individuals from the General Population. Clin. Chem. 2015, 61, 533-543. [CrossRef]

11. Elshazly, M.B.; Mani, P.; Nissen, S.; Brennan, D.M.; Clark, I.D.; Martin, S.; Jones, S.R.; Quispe, R.; Donnellan, E.; Nicholls, S.J.; et al. Remnant cholesterol, coronary atheroma progression and clinical events in statin-treated patients with coronary artery disease. Eur. J. Prev. Cardiol. 2020, 27, 1091-1100. [CrossRef]

12. Lin, A.; Nerlekar, N.; Rajagopalan, A.; Yuvaraj, J.; Modi, R.; Mirzaee, S.; Munnur, R.K.; Seckington, M.; Doery, J.C.; Seneviratne, S.; et al. Remnant cholesterol and coronary atherosclerotic plaque burden assessed by computed tomography coronary angiography. Atherosclerosis 2019, 284, 24-30. [CrossRef]

13. Masuda, D.; Yamashita, S. Postprandial Hyperlipidemia and Remnant Lipoproteins. J. Atheroscler. Thromb. 2017, 24, 95-109. [CrossRef]

14. Varbo, A.; Nordestgaard, B.G. Remnant lipoproteins. Curr. Opin. Lipidol. 2017, 28, 300-307. [CrossRef]

15. Li, Z.; Guo, X.; Zheng, L.; Yang, H.; Sun, Y. Grim status of hypertension in rural China: Results from Northeast China Rural Cardiovascular Health Study 2013. J. Am. Soc. Hypertens. 2015, 9, 358-364. [CrossRef] [PubMed]

16. Hypertension Control: Report of a WHO Expert Committee; World Health Organization Technical Report Series; WHO: Geneva, Switzerland, 1996; Volume 862, pp. 1-83.

17. Cao, Y.-X.; Zhang, H.-W.; Jin, J.-L.; Liu, H.-H.; Zhang, Y.; Gao, Y.; Guo, Y.-L.; Wu, N.-Q.; Hua, Q.; Li, Y.-F.; et al. The longitudinal association of remnant cholesterol with cardiovascular outcomes in patients with diabetes and pre-diabetes. Cardiovasc. Diabetol. 2020, 19, 104. [CrossRef] [PubMed]

18. World Health Organization. Definition and Diagnosis of Diabetes Mellitus and Intermediate Hyperglycemia: Report of a WHO/IDF Consultation; WHO: Geneva, Switzerland, 2006; pp. 1-3.

19. Levey, A.S.; Coresh, J.; Greene, T.; Stevens, L.A.; Zhang, Y.; Hendriksen, S.; Kusek, J.W.; Van Lente, F. Using Standardized Serum Creatinine Values in the Modification of Diet in Renal Disease Study Equation for Estimating Glomerular Filtration Rate. Ann. Intern. Med. 2006, 145, 247-254. [CrossRef]

20. Chuang, S.-Y.; Chen, J.-H.; Yeh, W.-T.; Wu, C.-C.; Pan, W.-H. Hyperuricemia and increased risk of ischemic heart disease in a large Chinese cohort. Int. J. Cardiol. 2012, 154, 316-321. [CrossRef] [PubMed]

21. Zhao, D.; Liu, J.; Wang, W.; Zeng, Z.; Cheng, J.; Liu, J.; Sun, J.; Wu, Z. Epidemiological transition of stroke in china: Twenty-one-year observational study from the sino-monica-beijing project. Stroke 2008, 39, 1668-1674. [CrossRef]

22. Gaye, B.; Canonico, M.; Perier, M.-C.; Samieri, C.; Berr, C.; Dartigues, J.-F.; Tzourio, C.; Elbaz, A.; Empana, J.-P. Ideal Cardiovascular Health, Mortality, and Vascular Events in Elderly Subjects. J. Am. Coll. Cardiol. 2017, 69, 3015-3026. [CrossRef] [PubMed]

23. Durrleman, S.; Simon, R. Flexible regression models with cubic splines. Stat. Med. 1989, 8, 551-561. [CrossRef]

24. Wu, Y.; Zhang, D.; Kang, S. Physical activity and risk of breast cancer: A meta-analysis of prospective studies. Breast Cancer Res. Treat. 2012, 137, 869-882. [CrossRef]

25. Lee, D.H.; Keum, N.; Hu, F.B.; Orav, E.J.; Rimm, E.B.; Willett, W.C.; Giovannucci, E.L. Predicted lean body mass, fat mass, and all cause and cause specific mortality in men: Prospective US cohort study. BMJ 2018, 362, k2575. [CrossRef]

26. Varbo, A.; Benn, M.; Tybjærg-Hansen, A.; Nordestgaard, B.G. Elevated Remnant Cholesterol Causes Both Low-Grade Inflammation and Ischemic Heart Disease, Whereas Elevated Low-Density Lipoprotein Cholesterol Causes Ischemic Heart Disease Without Inflammation. Circulation 2013, 128, 1298-1309. [CrossRef] [PubMed]

27. Varbo, A.; Benn, M.; Nordestgaard, B.G. Remnant cholesterol as a cause of ischemic heart disease: Evidence, definition, measurement, atherogenicity, high risk patients, and present and future treatment. Pharmacol. Ther. 2014, 141, 358-367. [CrossRef] [PubMed]

28. Jørgensen, A.B.; Frikke-Schmidt, R.; West, A.S.; Grande, P.; Nordestgaard, B.G.; Tybjærg-Hansen, A. Genetically elevated nonfasting triglycerides and calculated remnant cholesterol as causal risk factors for myocardial infarction. Eur. Heart J. 2013, 34, 1826-1833. [CrossRef] [PubMed]

29. Varbo, A.; Freiberg, J.J.; Nordestgaard, B.G. Remnant Cholesterol and Myocardial Infarction in Normal Weight, Overweight, and Obese Individuals from the Copenhagen General Population Study. Clin. Chem. 2018, 64, 219-230. [CrossRef]

30. Jepsen, A.-M.K.; Langsted, A.; Varbo, A.; Bang, L.E.; Kamstrup, P.R.; Nordestgaard, B.G. Increased Remnant Cholesterol Explains Part of Residual Risk of All-Cause Mortality in 5414 Patients with Ischemic Heart Disease. Clin. Chem. 2016, 62, 593-604. [CrossRef] 
31. Vallejo-Vaz, A.J.; Fayyad, R.; Boekholdt, M.; Hovingh, G.K.; Kastelein, J.J.; Melamed, S.; Barter, P.; Waters, D.D.; Ray, K.K. Triglyceride-Rich Lipoprotein Cholesterol and Risk of Cardiovascular Events Among Patients Receiving Statin Therapy in the TNT Trial. Circulation 2018, 138, 770-781. [CrossRef] [PubMed]

32. Andersson, C.; Vasan, R.S. Epidemiology of cardiovascular disease in young individuals. Nat. Rev. Cardiol. 2018, 15, 230-240. [CrossRef]

33. Batalla, A.; Reguero, J.R.; Hevia, S.; Cubero, G.I.; Cortina, A. Mild hypercholesterolemia and premature heart disease. J. Am. Coll. Cardiol. 2001, 37, 331-332. [CrossRef]

34. Khot, U.N.; Khot, M.B.; Bajzer, C.T.; Sapp, S.K.; Ohman, E.M.; Brener, S.J.; Ellis, S.G.; Lincoff, A.M.; Topol, E. Prevalence of Conventional Risk Factors in Patients with Coronary Heart Disease. JAMA 2003, 290, 898-904. [CrossRef]

35. Yang, Q.; Cogswell, M.E.; Flanders, W.D.; Hong, Y.; Zhang, Z.; Loustalot, F.; Gillespie, C.; Merritt, R.; Hu, F.B. Trends in Cardiovascular Health Metrics and Associations With All-Cause and CVD Mortality Among US Adults. JAMA 2012, 307, 1273-1283. [CrossRef]

36. Laslett, L.J.; Alagona, P., Jr.; Clark, B.A., 3rd; Drozda, J.P., Jr.; Saldivar, F.; Wilson, S.R.; Poe, C.; Hart, M. The worldwide environment of cardiovascular disease: Prevalence, diagnosis, therapy, and policy issues: A report from the american college of cardiology. J. Am. Coll. Cardiol. 2012, 60, S1-S49. [CrossRef]

37. Spring, B.; Moller, A.C.; Colangelo, L.A.; Siddique, J.; Roehrig, M.; Daviglus, M.L.; Polak, J.F.; Reis, J.P.; Sidney, S.; Liu, K. Healthy lifestyle change and subclinical atherosclerosis in young adults: Coronary Artery Risk Development in Young Adults (CARDIA) study. Circulation 2014, 130, 10-17. [CrossRef]

38. Hobbs, F.D.R.; Jukema, J.W.; Da Silva, P.M.; McCormack, T.; Catapano, A.L. Barriers to cardiovascular disease risk scoring and primary prevention in Europe. QJM 2010, 103, 727-739. [CrossRef]

39. Lloyd-Jones, D.M. Cardiovascular risk prediction: Basic concepts, current status, and future directions. Circulation 2010, 121, 1768-1777. [CrossRef]

40. Garshick, M.S.; Vaidean, G.D.; Vani, A.; Underberg, J.A.; Newman, J.D.; Berger, J.; Fisher, E.A.; Gianos, E. Cardiovascular Risk Factor Control and Lifestyle Factors in Young to Middle-Aged Adults with Newly Diagnosed Obstructive Coronary Artery Disease. Cardiology 2019, 142, 83-90. [CrossRef] [PubMed]

41. Kannel, W.B.; McGee, D.L. Diabetes and cardiovascular disease. The framingham study. JAMA 1979, 241, 2035-2038. [CrossRef]

42. Schaefer, E.J.; McNamara, J.R.; Shah, P.K.; Nakajima, K.; Cupples, L.A.; Ordovas, J.; Wilson, P.W. Elevated Remnant-Like Particle Cholesterol and Triglyceride Levels in Diabetic Men and Women in the Framingham Offspring Study. Diabetes Care 2002, 25, 989-994. [CrossRef]

43. Jørgensen, P.G.; Jensen, M.T.; Biering-Sørensen, T.; Mogelvang, R.; Galatius, S.; Fritz-Hansen, T.; Rossing, P.; Vilsbøll, T.; Jensen, J.S. Cholesterol remnants and triglycerides are associated with decreased myocardial function in patients with type 2 diabetes. Cardiovasc. Diabetol. 2016, 15, 137. [CrossRef]

44. Xu, Y.; Wang, L.; He, J.; Bi, Y.; Li, M.; Wang, T.; Wang, L.; Jiang, Y.; Dai, M.; Lu, J.; et al. Prevalence and Control of Diabetes in Chinese Adults. JAMA 2013, 310, 948-959. [CrossRef]

45. Castañer, O.; Pintó, X.; Subirana, I.; Amor, A.J.; Ros, E.; Hernáez, Á.; Martínez-González, M.Á.; Corella, D.; Salas-Salvadó, J.; Estruch, R.; et al. Remnant Cholesterol, Not LDL Cholesterol, Is Associated With Incident Cardiovascular Disease. J. Am. Coll. Cardiol. 2020, 76, 2712-2724. [CrossRef]

46. Qin, Z.; Zhou, K.; Li, Y.-P.; Wang, J.-L.; Cheng, W.-J.; Hu, C.-P.; Shi, C.; He, H.; Zhou, Y.-J. Remnant lipoproteins play an important role of in-stent restenosis in type 2 diabetes undergoing percutaneous coronary intervention: A single-centre observational cohort study. Cardiovasc. Diabetol. 2019, 18, 1-9. [CrossRef]

47. Grundy, S.M.; Stone, N.J.; Bailey, A.L.; Beam, C.; Birtcher, K.K.; Blumenthal, R.S.; Braun, L.T.; de Ferranti, S.; Faiella-Tommasino, J.; Forman, D.E.; et al. 2018 aha/acc/aacvpr/aapa/abc/acpm/ada/ags/apha/aspc/nla/pcna guideline on the management of blood cholesterol: A report of the american college of cardiology/american heart association task force on clinical practice guidelines. J. Am. Coll. Cardiol. 2019, 73, e285-e350. [CrossRef]

48. Cosentino, F.; Grant, P.J.; Aboyans, V.; Bailey, C.J.; Ceriello, A.; Delgado, V.; Federici, M.; Filippatos, G.; Grobbee, E.D.; Hansen, T.B.; et al. 2019 ESC Guidelines on diabetes, pre-diabetes, and cardiovascular diseases developed in collaboration with the EASD. Eur. Heart J. 2019, 41, 255-323. [CrossRef]

49. Hansen, S.E.; Madsen, C.M.; Varbo, A.; Nordestgaard, B.G. Low-Grade Inflammation in the Association between Mild-toModerate Hypertriglyceridemia and Risk of Acute Pancreatitis: A Study of More Than 115000 Individuals from the General Population. Clin. Chem. 2019, 65, 321-332. [CrossRef]

50. Balling, M.; Langsted, A.; Afzal, S.; Varbo, A.; Smith, G.D.; Nordestgaard, B.G. A third of nonfasting plasma cholesterol is in remnant lipoproteins: Lipoprotein subclass profiling in 9293 individuals. Atherosclerosis 2019, 286, 97-104. [CrossRef]

51. Twickler, T.B.; Dallinga-Thie, G.M.; Cohn, J.S.; Chapman, M.J. Elevated remnant-like particle cholesterol concentration: A characteristic feature of the atherogenic lipoprotein phenotype. Circulation 2004, 109, 1918-1925. [CrossRef]

52. Shin, H.K.; Kim, Y.K.; Kim, K.Y.; Lee, J.H.; Hong, K.W. Remnant lipoprotein particles induce apoptosis in endothelial cells by $\operatorname{nad}(\mathrm{p}) \mathrm{h}$ oxidase-mediated production of superoxide and cytokines via lectin-like oxidized low-density lipoprotein receptor-1 activation: Prevention by cilostazol. Circulation 2004, 109, 1022-1028. [CrossRef]

53. Nakajima, K.; Nakano, T.; Tanaka, A. The oxidative modification hypothesis of atherosclerosis: The comparison of atherogenic effects on oxidized LDL and remnant lipoproteins in plasma. Clin. Chim. Acta 2006, 367, 36-47. [CrossRef] 
54. Liu, L.; Wen, T.; Zheng, X.-Y.; Yang, D.-G.; Zhao, S.-P.; Xu, D.-Y.; Lü, G.-H. Remnant-like particles accelerate endothelial progenitor cells senescence and induce cellular dysfunction via an oxidative mechanism. Atherosclerosis 2009, 202, 405-414. [CrossRef]

55. Kitulwatte, I.D.; Pollanen, M.S. A Comparative Study of Coronary Atherosclerosis in Young and Old. Am. J. Forensic Med. Pathol. 2015, 36, 323-326. [CrossRef] [PubMed]

56. Kostara, C.; Papathanasiou, A.; Psychogios, N.; Cung, M.T.; Elisaf, M.; Goudevenos, J.; Bairaktari, E.T. NMR-Based Lipidomic Analysis of Blood Lipoproteins Differentiates the Progression of Coronary Heart Disease. J. Proteome Res. 2014, 13, 2585-2598. [CrossRef]

57. Mazzone, T.; Chait, A.; Plutzky, J. Cardiovascular disease risk in type 2 diabetes mellitus: Insights from mechanistic studies. Lancet 2008, 371, 1800-1809. [CrossRef] 